\title{
Gas permeability measurements from pressure pulse decay laboratory data using pseudo-pressure and pseudo-time transformations
}

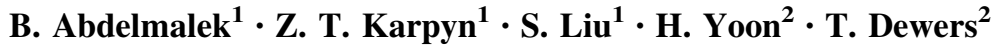

Received: 17 December 2016/Accepted: 23 July 2017/Published online: 4 August 2017

(c) The Author(s) 2017. This article is an open access publication

\begin{abstract}
This paper presents a pseudo-pressure and pseudo-time straight-line approach to interpret laboratory pulse decay data in order to estimate rock core permeability using gas as the pore fluid. The implementation of the straight-line approach provides a practical method to estimate gas permeability from experimental data, as long as changes in gas viscosity and compressibility are negligible. On the other hand, pseudo-pressure and pseudo-time allow the transformation of the compressible flow equation from its highly nonlinear form to a quasi-linear partial differential equation, where changes in gas viscosity, gas compressibility and compressibility factor are accounted for. The purpose of this work is to combine both, pseudofunctions and the straight-line approach to estimate gas permeability from pressure pulse laboratory data with a more rigorous treatment of gas properties. Five pulse decay experiments were performed in Marcellus shale cores at pore pressures ranging from 130 to $700 \mathrm{psi}$ in a triaxial cell to estimate permeability and porosity of ultra-low permeability cores. The experiments were made in an increasing order of equilibration pressure starting from 130 until $700 \mathrm{psi}$, and vertical and radial stresses kept constant at 1500 psi. Permeability estimates were compared against the $P^{2}$-approach to show the validity of the proposed method at low gas pressures.
\end{abstract}

Z. T. Karpyn

ZKarpyn@psu.edu

1 John and Willie Leone Family Department of Energy and Mineral Engineering, EMS Energy Institute, The Pennsylvania State University, 151 Hosler Bldg, University Park, PA 16802, USA

2 Geoscience Research and Applications, Sandia National

Laboratories, Albuquerque, NM, USA
Keywords Gas permeability · Pulse decay · Pseudopressure $\cdot$ Pseudo-time

\section{List of symbols}

A Cross-sectional area of the core $\left(\mathrm{in}^{2}\right)$

$c_{\text {gui }} \quad$ Gas compressibility in the upstream at the value of initial upstream pressure right after applying the pulse

$c_{\mathrm{gdi}}$ Gas compressibility in the downstream at a pressure value of equilibration pore pressure $\left(\mathrm{psi}^{-1}\right)$

$c_{\mathrm{gp}} \quad$ Gas compressibility at initial pore pressure $\left(\mathrm{psi}^{-1}\right)$

$c_{\mathrm{tp}} \quad$ Total compressibility at initial pore pressure $\left(\mathrm{psi}^{-1}\right)$

$f_{1} \quad$ "Mass flow correction factor," from Jones (1997)

$k \quad$ Permeability (md)

$L \quad$ Length of the core (in)

$m_{1} \quad$ The slope of dimensionless pressure versus time data $\left(\mathrm{s}^{-1}\right)$

$P \quad$ Pressure (psia)

$P_{\mathrm{d}} \quad$ Downstream pressure (psi)

$\left(P_{\text {eq }}\right)_{\mathrm{p}} \quad$ Equilibration pseudo-pressure $\left(\mathrm{psia}^{2} / \mathrm{cp}\right)$

$P_{\mathrm{o}} \quad$ Initial pore pressure (psia)

$P_{\mathrm{p}} \quad$ Pseudo-pressure $\left(\mathrm{psia}^{2} / \mathrm{cp}\right)$

$P_{\mathrm{pd}} \quad$ Downstream pseudo-pressure $\left(\mathrm{psia}^{2} / \mathrm{cp}\right)$

$P_{\mathrm{pp}} \quad$ Pulse pseudo-pressure ( $\left.\mathrm{psia}^{2} / \mathrm{cp}\right)$

$P_{\mathrm{pu}} \quad$ Upstream pseudo-pressure $\left(\mathrm{psia}^{2} / \mathrm{cp}\right)$

$P_{\mathrm{u}} \quad$ Upstream pressure (psi)

$V_{\mathrm{u}} \quad$ Upstream volume $\left(\mathrm{in}^{3}\right)$

$V_{\mathrm{d}} \quad$ Downstream volume $\left(\mathrm{in}^{3}\right)$

$z \quad$ Compressibility factor (dimensionless)

$\alpha \quad$ Dimensionless time 
$\beta \quad$ Ratio of compressive storage in the sample to the compressive storage in the downstream volume (dimensionless)

$\gamma \quad$ Ratio of compressive storage in the downstream to the compressive storage in the upstream (dimensionless)

$\theta_{\mathrm{m}} \quad$ Roots of the equation

$\mu \quad$ Viscosity (cp)

$\mu_{\mathrm{ui}} \quad$ Viscosity of the fluid in the upstream at time $=0$ (cp)

$\mu_{\mathrm{di}} \quad$ Viscosity of the fluid in the downstream at time $=0(\mathrm{cp})$

$\mu_{\mathrm{p}} \quad$ Viscosity at initial pore pressure (cp)

$\phi \quad$ Porosity, fraction

\section{Introduction}

Laboratory measurements of permeability in tight and ultra-tight rocks are of great importance in view of increased interest in the characterization of unconventional oil and gas reservoirs. However, most standard methods of permeability measurement suffer from either theoretical or practical shortcomings. For example, permeability measurement for tight rocks using a steady-state method is time-consuming (Zamirian et al. 2014), as a steady flow can easily take weeks to be established in tight rocks like shale and coal. Since the steady-state methods are slow to estimate permeability, other methods have been introduced to measure the permeability of tight rocks. Some of the relatively recent methods are based on pressure pulse data (Brace et al. 1968; Hsieh et al. 1981; Dicker and Smits 1988; Jones 1997), crushed sample technique (Cui et al. 2009), pore pressure oscillations (Fischer 1992) and complex pore pressure transients (Boitnott 1997).

Each of the aforementioned recent methods has its advantage and disadvantage. First, crushed sample method is the simplest method where only two chambers of known volumes are needed and gas is allowed to expand into the crushed sample chamber from a reference chamber (Cui et al. 2009; Zamirian et al. 2014). Using pressure and time data, one can calculate the permeability. However, crushed samples do not allow for permeability measurements under variable stress conditions. Second, the pulse decay technique requires sending a pressure pulse from the upstream volume, which will permeate through the sample to a downstream volume (Brace et al. 1968; Hsieh et al. 1981; Dicker and Smits 1988; Jones 1997). The pressure decay response is continuously recorded with time, and then, sample permeability is estimated by interpretation of pressure decay curve. The benefit from the pulse decay is the time reduction in obtaining permeability of tight samples. However, as will be discussed thoroughly in this paper, the current models do not accurately deal with pulse decay experiments at pressure ranges when gas behaves non-ideally. Third, pore pressure oscillations method was proposed in order to tackle the problem of getting noisy signal from the pulse decay technique. In this method, sinusoidal pore pressure pulse is sent from the upstream of the sample and based on the retardation of the signal and phase change at the downstream, permeability can be calculated (Fischer 1992; Jin et al. 2015). However, recent pressure transducers do not cause noisy signals that require a new modification on enhancing the signal, especially when the signal is clear from a simpler measurement technique like the pulse decay. Finally, complex pore pressure oscillations method is similar to the pore pressure oscillations methods, but instead of sending a sinusoidal pore pressure pulse from the upstream, different pore pressure pulses that are more distinct are sent (Boitnott 1997). This method has an advantage over the pore pressure oscillations that it can take measurements for high permeability samples; however, it still adds complexity that is unneeded as the same measurements can be taken in a simpler manner. Therefore, it was found in this work that the pulse decay is a method that offers a good degree of accuracy and speed with the minimum amount of work needed.

The data from the pulse decay experiment can be manipulated in two different ways: a straight-line method (Brace et al. 1968; Dicker and Smits 1988; Jones 1997) and type curve matching (Hsieh et al. 1981; Neuzil et al. 1981; Haskett et al. 1988; Kamath et al. 1992). "Straight-line" method requires the determination of the slope of the straight-line in a semilog plot of pressure decay versus time. Based on the slope, the permeability can be estimated since it is the only unknown parameter in the analysis. On the other hand, type curve matching is to compare theoretical curves that are generated from fluid flow mathematical models in terms of dimensionless parameters. Once the match points between theoretical and experimental data are identified, both permeability and porosity can be determined. Regardless of the types of manipulation, one has to choose the most appropriate mathematical model or governing equation that can represent the fluid flow during the experiment, e.g., incompressible, slightly compressible or compressible flow.

The pulse decay approaches can be categorized based on the governing equations and methods of data interpretation. First, Brace et al. (1968) used the incompressible flow equation as a governing equation and the straight-line method as a data manipulation method. However, the incompressible flow model cannot capture the physics of compressible fluids; Brace et al. (1968) method may give correct permeability trends instead of correct permeability values, for example, detecting changes in permeability as function of stress conditions, water content and 
heterogeneity. Second, Hsieh et al. (1981), Neuzil et al. (1981) used the slightly compressible flow equation as the governing equation and type curve matching as a method of data manipulation. It should be mentioned that Hsieh et al. (1981) work presented the generalized solutions and used the type curve. The only limitation with their work is that the slightly compressible flow equation is unable to analyze gas flow problems at some pressure ranges. Third, Dicker and Smits (1988) and Jones (1997) methods do represent the straight-line methods with the slightly compressible equation. The straight-line approach reduces the amount of work done; however, the slightly compressible equation, as mentioned previously, does have limitations when dealing with gases. Finally, Haskett et al. (1988) were the first to propose the use of the pseudo-pressure and pseudo-time approach to analyze the pulse decay experiments. The advantage of the pseudo-pressure approach is that changes in viscosity and compressibility during the experiment can be accounted for in the governing equation. However, Haskett et al. (1988) method had two limitations. First, the method requires the conversion of the differential pressure data to pseudo-pressures, which can be problematic due to the nonlinear relationship between pressure and pseudo-pressure at low pressures. Second, the method requires developing a computer code in order to do the type curve matching automatically.

Among the methods described, the pseudo-pressure and pseudo-time approach would be the most accurate way for analyzing the pulse decay experiments with gases because the pseudo-pressure approach is based on the compressible flow equation; therefore, the method can accurately describe the behavior of gases in general. The only method that attempted to apply the pseudo-pressure approach (Haskett et al. 1988) has some limitations. Those limitations, as mentioned previously, had to do with converting the difference in pressure between the upstream and downstream to pseudo-pressures and the significant amount of work for the type curve matching. Therefore, there is a need to accurately and efficiently treat compressible fluids pulse decay experiments to estimate the permeability. In this work, a pseudo-pressure and pseudo-time straight-line approach is proposed to resolve the treatment of gas viscosity and compressibility in the estimation of gas permeability from pulse decay experiments.

\section{Derivation of the governing equation}

In this work, fluid flow in porous media is described by the one-dimensional compressible flow equation in its linearized form using the pseudo-pressure and pseudo-time (Eq. 1). The notation used in the following equations is given at the end of the manuscript. $\frac{\partial^{2} P_{\mathrm{p}}}{\partial x^{2}}=\frac{\phi \overline{\mu c_{\mathrm{t}}}}{k} \frac{\partial P_{\mathrm{p}}}{\partial t_{\mathrm{p}}}$

where $P_{\mathrm{p}}$ is the pseudo-pressure, $\bar{\mu}$ is the mean gas viscosity, $\overline{c_{\mathrm{t}}}$ is the mean total compressibility, $t_{\mathrm{p}}$ is the pseudo-time, and $k$ is the permeability. The pseudo-pressure and pseudo-time are defined as shown in Eqs. (2) and (3).

$P_{\mathrm{p}}=2 \int_{P_{\mathrm{o}}}^{P} \frac{P}{\mu \mathrm{Z}} \mathrm{d} p$

$t_{\mathrm{p}}=\overline{\mu c} \int_{t_{\mathrm{o}}}^{t} \frac{1}{\mu c_{\mathrm{t}}} \mathrm{d} t$

In order to solve the governing equation for the pressure pulse decay experiment, the boundary conditions are specified as follows:

$P_{\mathrm{p}}(x, 0)=\left(P_{\mathrm{eq}}\right)_{\mathrm{p}}$ for $0<x<L$

$P_{\mathrm{p}}(0, t)=P_{\mathrm{pd}}(t) \quad$ for $\quad t>0$

$P_{\mathrm{p}}(L, t)=P_{\mathrm{pu}}(t)$ for $t>0$

$\frac{\mu_{\mathrm{ui}} c_{\mathrm{gui}} V_{\mathrm{u}}}{k A} \frac{\partial P_{\mathrm{pu}}}{\partial t}-\left(\frac{\partial P_{\mathrm{p}}}{\partial x}\right)_{x=L}=0$ for $t>0$

$P_{\mathrm{Pu}}(0)=P_{\mathrm{pp}} \quad$ for $\quad t=0$

$\frac{\mu_{\mathrm{di}} c_{\mathrm{gdi}} V_{\mathrm{d}}}{k A} \frac{\partial P_{\mathrm{pd}}}{\partial t}-\left(\frac{\partial P_{\mathrm{p}}}{\partial x}\right)_{x=0}=0$ for $t>0$

$P_{\mathrm{Pd}}(0)=0$ for $t=0$

Equation (4) states that the initial pseudo-pressure $P_{\mathrm{p}}(x, 0)$ is equal to the equilibrated pseudo-pressure $\left(P_{\text {eq }}\right)_{\mathrm{p}}$. Equations (5-6) describe the boundary conditions of the pseudopressure at $x=0$ (downstream) and $x=L$ (upstream), respectively. Here subscripts, $d$ and $u$, represent the downstream and upstream, respectively. At $x=0$ and $x=L$, the pseudo-pressures are the downstream $\left(P_{\mathrm{pd}}(t)\right)$ and upstream $\left(P_{\mathrm{pu}}(t)\right)$ pseudo-pressure, respectively. Equation (7) represents the mass balance at $x=L$ where $\mu_{\mathrm{ui}}$ is the fluid viscosity used in the experiment in the upstream reservoir at $t=0, c_{\text {gui }}$ is the fluid compressibility in the upstream reservoir at time $=0, V_{\mathrm{u}}$ is the upstream volume, and $A$ is the cross-sectional area of the sample that is perpendicular to the flow. Similarly, Eq. (9) represents the mass balance in the downstream end of the sample. Equations (8) and (10) are initial conditions.

The governing Eq. (1) with the boundary conditions (310) can be solved by the Laplace transform method. The solutions for dimensionless pseudo-pressures for upstream and downstream are given in Eqs. (11) and (12), which were first developed by Hsieh et al. (1981) and then Haskett et al. (1988) adapted them for the pseudo-pressure approach. 


$$
\begin{aligned}
& \frac{P_{\mathrm{pu}}}{P_{\mathrm{pp}}}=\frac{1}{1+\beta+\gamma} \\
& +2 \sum_{m=1}^{\infty}\left[\left(\frac{\left(\beta+\frac{\gamma^{2} \theta_{m}^{2}}{\beta}\right)}{\frac{\gamma^{2} \theta_{m}^{4}}{\beta^{2}}+\frac{\left(\gamma^{2} \beta+\gamma^{2}+\gamma+\beta\right) \theta_{m}^{2}}{\beta}+\left(\beta^{2}+\gamma \beta+\beta\right)}\right) \mathrm{e}^{-\alpha \theta_{m}^{2}}\right]
\end{aligned}
$$

$$
\frac{P_{\mathrm{pd}}}{P_{\mathrm{pp}}}=\frac{1}{1+\beta+\gamma}
$$$$
+2 \sum_{m=1}^{\infty}\left[\left(\frac{\left(\beta-\frac{\gamma \theta_{m}^{2}}{\beta}\right)}{\left[\frac{\gamma^{2} \theta_{m}^{4}}{\beta^{2}}+\frac{\left(\gamma^{5} \beta+\gamma^{2}+\gamma+\beta\right) \theta_{m}^{2}}{\beta}+\left(\beta^{2}+\gamma \beta+\beta\right)\right] \cos \theta_{m}}\right) \mathrm{e}^{-\alpha \theta_{m}^{2}}\right]
$$

In the above-generalized solutions, three dimensionless variables $(\alpha, \beta$ and $\gamma)$ are defined: $\alpha$ is the dimensionless time, $\gamma$ is the ratio of the compressive storage of the downstream to the compressive storage of the upstream volume, and $\beta$ is the ratio of the compressive storage of sample to the compressive storage of upstream. The compressive storage of upstream, downstream or sample is defined as the change in the volume of a fluid per unit change in pressure for the upstream, downstream or sample, respectively. These dimensionless variables are given by Eqs. (13), (14) and (15):

$$
\begin{aligned}
& \alpha=\frac{k t}{94838.5 \phi \overline{\mu c}_{\mathrm{t}} L^{2}} \\
& \gamma=\frac{V_{\mathrm{d}}}{V_{\mathrm{u}}} \\
& \beta=\frac{V_{\mathrm{p}} c_{\mathrm{tp}}}{V_{\mathrm{u}} c_{\text {gui }}}
\end{aligned}
$$

Additional parameter $\theta_{\mathrm{m}}$ in Eqs. (11-12) solution is defined as the roots of the following equation:

$\tan \theta_{m}=\frac{(1+\gamma) \theta_{m}}{\frac{\gamma \theta_{m}^{2}}{\beta}-\beta}$

Figure 1 shows an example of the pseudo-pressure type curves obtained for $\gamma=1$ where the compressive storage of downstream and upstream is the same. The $\beta$ values vary between 0.001 and 10 , because the type curves will overlap for $\beta$ values below 0.001 and above 10 (Hsieh et al. 1981). In this work, the $\theta_{\mathrm{m}}$ in Eq. 16 is numerically computed using the Newton-Raphson method as presented in "Appendix 1." Finally, the dimensionless time $(\alpha)$ is increasingly changed until upstream and downstream pressures meet at $\frac{P_{\mathrm{pu}}}{P_{\mathrm{pp}}}=\frac{P_{\mathrm{pd}}}{P_{\mathrm{pp}}}=\frac{1}{1+\beta+\gamma}$. The family of type curves in Fig. 1 also matches those constructed by Hsieh et al. (1981).

Another general solution for the pulse decay problem was introduced by Dicker and Smits (1988) where

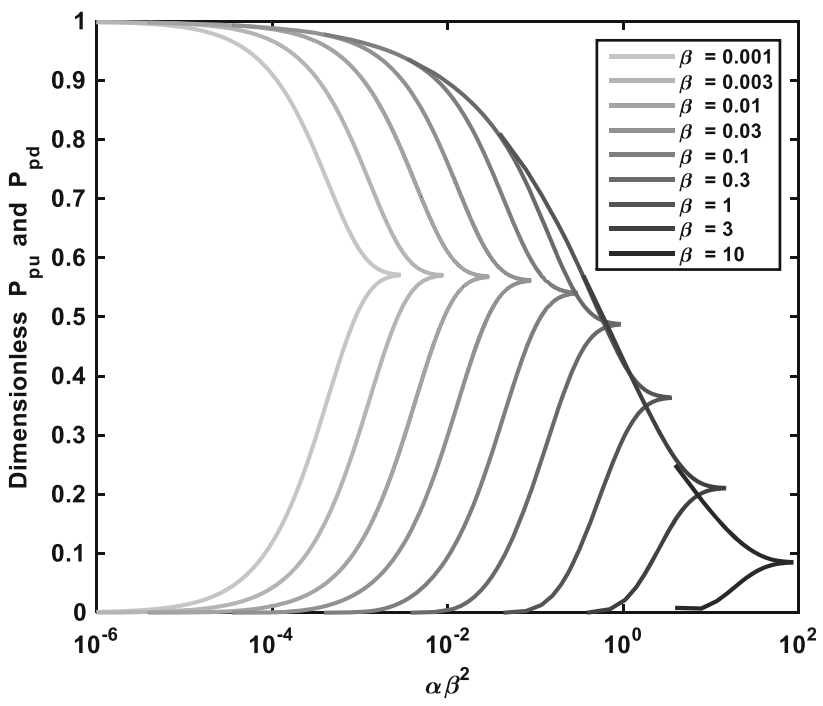

Fig. 1 Family of type curves for $\gamma=1$ and $\beta$ between 0.001 and 10 constructed using pseudo-pressure type curves

dimensionless pressure drop is employed instead of the dimensionless upstream and downstream in the type curve approach. In addition, the terms " $a$ " and " $b$ " were used instead of $\gamma$ and $\beta$ terms. Due to its mathematical form, this approach is referred to as the straight-line approach. The Dicker and Smits (1988) solution is as follows:

$$
\begin{aligned}
\Delta P_{\mathrm{D}}= & 2 \sum_{m=1}^{\infty}\left(\frac{a\left(b^{2}+\theta_{m}^{2}\right)-(-1)^{m} b \sqrt{\left(a^{2}+\theta_{m}^{2}\right)\left(b^{2}+\theta_{m}^{2}\right)}}{\theta_{m}^{2}\left(\theta_{m}^{2}+a+a^{2}+b+b^{2}\right)+a b(a+b+a b)}\right) \\
& \times \exp \left(-\theta_{m}^{2} \alpha\right)
\end{aligned}
$$

where the compressive storage ratios " $a$ " and " $b$ " in the Dicker and Smits (1988)'s solution were defined as:

$a=\frac{V_{\mathrm{p}}\left(c_{\mathrm{g}}+c_{\mathrm{pv}}\right)}{V_{1}\left(c_{\mathrm{g}}+c_{\mathrm{v}_{1}}\right)}$

$b=\frac{V_{\mathrm{p}}\left(c_{\mathrm{g}}+c_{\mathrm{pv}}\right)}{V_{2}\left(c_{\mathrm{g}}+c_{\mathrm{v}_{1}}\right)}$

The relation of the compressive storage terms in these two solutions is given by:

$a=\beta$

$b=\frac{\beta}{\gamma}$

In addition, Eq. (16) can be expressed in terms of " $a$ " and " $b$ ":

$\tan \theta_{m}=\frac{(a+b) \theta}{\theta^{2}-a b}$

Considering the first term of the series in Eq. (17) with the first root of Eq. (22), this equation becomes: 
Fig. 2 Schematic diagram of triaxial cell and experimental assembly

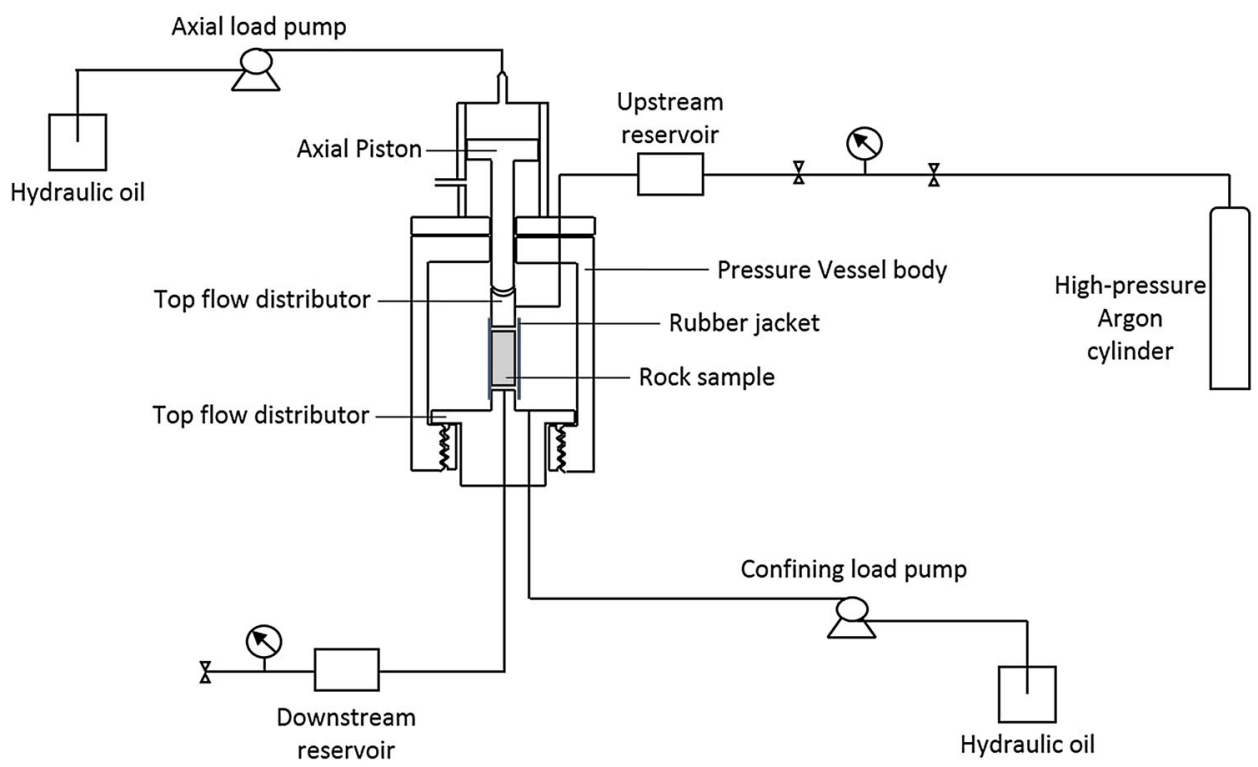

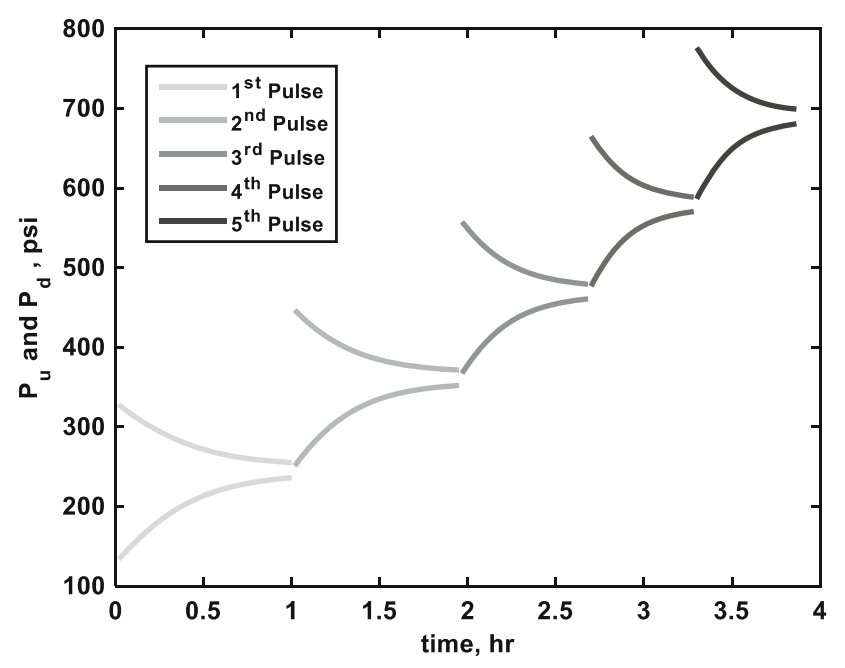

Fig. 3 Pressure history of pulse decay experiment in Marcellus shale core

$$
\begin{aligned}
\Delta P_{\mathrm{D}}= & 2\left(\frac{a\left(b^{2}+\theta_{1}^{2}\right)-(-1)^{m} b \sqrt{\left(a^{2}+\theta_{1}^{2}\right)\left(b^{2}+\theta_{1}^{2}\right)}}{\theta_{1}^{2}\left(\theta_{1}^{2}+a+a^{2}+b+b^{2}\right)+a b(a+b+a b)}\right) \\
& \times \exp \left(-\theta_{1}^{2} \alpha\right)
\end{aligned}
$$

If the natural $\log$ is taken for both sides for Eq. (23), we get:

$\ln \left(\Delta P_{\mathrm{D}}\right)=b-\theta_{1}^{2} \alpha$

where

$$
\begin{aligned}
b & =\text { intercept } \\
& =\ln \left(2\left(\frac{a\left(b^{2}+\theta_{1}^{2}\right)-(-1)^{m} b \sqrt{\left(a^{2}+\theta_{1}^{2}\right)\left(b^{2}+\theta_{1}^{2}\right)}}{\theta_{1}^{2}\left(\theta_{1}^{2}+a+a^{2}+b+b^{2}\right)+a b(a+b+a b)}\right)\right)
\end{aligned}
$$

The straight-line analysis can be performed using this equation:

$\ln \left(\Delta P_{\mathrm{D}}\right)=b-\frac{k \theta_{1}^{2}}{94838.5 \phi \overline{\mu c}_{\mathrm{t}} L^{2}} \times t$

From the slope of a straight-line drawn between $\Delta P_{\mathrm{D}}$ and $\mathrm{t}$

\begin{tabular}{|c|c|c|c|c|c|c|c|c|c|}
\hline Equilibration pressure (psi) & ${\overline{c_{g}}}_{\text {up }}$ & ${\overline{c_{g}}}_{\text {down }}$ & $a$ & $b$ & $\gamma$ & $\beta$ & $\theta_{1}$ & Slope, Fig. 5 & Slope, Fig. 6 \\
\hline 246.5 & $3.50 \mathrm{E}-03$ & $5.30 \mathrm{E}-03$ & 0.23 & 0.16 & 1.43 & 0.23 & 0.61 & $-5.0 \mathrm{E}-04$ & $-6.1 \mathrm{E}-04$ \\
\hline 362.5 & $2.50 \mathrm{E}-03$ & $3.30 \mathrm{E}-03$ & 0.42 & 0.34 & 1.25 & 0.42 & 0.85 & $-5.7 \mathrm{E}-04$ & $-6.4 \mathrm{E}-04$ \\
\hline 470 & $2.00 \mathrm{E}-03$ & $2.40 \mathrm{E}-03$ & 0.69 & 0.61 & 1.14 & 0.69 & 1.08 & $-7.4 \mathrm{E}-04$ & $-7.7 \mathrm{E}-04$ \\
\hline 580 & $1.60 \mathrm{E}-03$ & $1.90 \mathrm{E}-03$ & 0.79 & 0.71 & 1.13 & 0.79 & 1.15 & $-9.9 \mathrm{E}-04$ & $-1.0 \mathrm{E}-03$ \\
\hline 696.5 & $1.35 \mathrm{E}-03$ & $1.90 \mathrm{E}-03$ & 0.77 & 0.68 & 1.12 & 0.77 & 1.13 & $-1.0 \mathrm{E}-03$ & $-1.0 \mathrm{E}-03$ \\
\hline
\end{tabular}
in Eq. (26), the permeability can be calculated if the porosity is determined independently.

Table 1 Model parameters obtained from five pulse decay experiments to estimate core permeability 

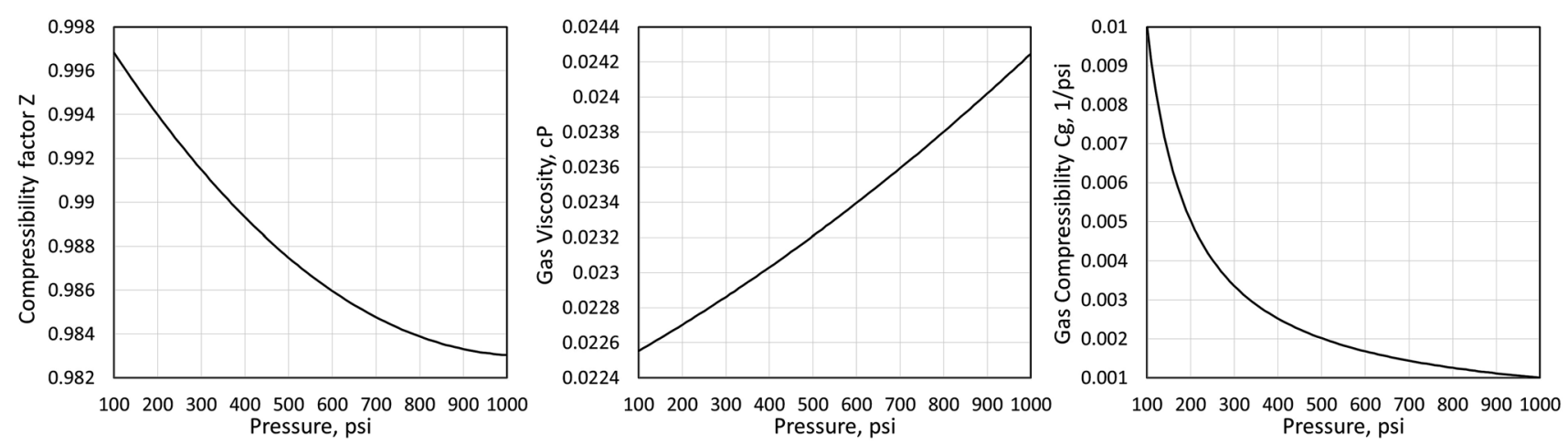

Fig. 4 Compressibility factor $Z$, gas viscosity and gas compressibility as a function of pressure

\section{Results and discussion}

Five pulse decay experiments were conducted on Marcellus shale cores at pore pressures ranging from 130 to 700 psi. The cores used were obtained from a Marcellus Shale well in Tioga county, Pennsylvania. In the five sequences, both vertical and radial stresses were kept constant at $1500 \mathrm{psi}$, as defined by instrumentation constraints. Figure 2 shows the experimental assembly used for these sequences. It consists of a triaxial cell, a Quizix pump, two omega pressure sensors, a data acquisition system (DAQ) and a high pressure Argon cylinder. Sample dimensions were $2.54 \mathrm{~cm}$ diameter and $5.715 \mathrm{~cm}$ length.

The experiment started by setting both vertical and radial stresses on the sample to 1500 psi of water using one Quizix pump by branching out the pump outlet to feed both the confining stress and axial load inlets as shown in Fig. 2. The pore pressure was equilibrated to 130.5 psi including the upstream and downstream volumes. Following pressure equilibration, a pressure pulse of an approximate $200 \mathrm{psi}$ was introduced from the upstream side of the system. The pressure response in both upstream and downstream volumes was recorded at a sampling rate of 1 pressure reading per $600 \mathrm{~ms}$ until upstream and downstream pressures equilibrate. In the same manner, four additional pulse decays were performed at increasingly higher upstream pressures, using similar pulse magnitudes of 200 psi. Figure 3 shows the sequence of the pulse decay sequences and the corresponding pressure profiles.

The model parameters used in the calculation of permeability using the proposed pseudo-pressure and pseudo-time straight-line approach and the $P^{2}$-approach are presented in Table 1 . Every row corresponds to one of the five sequences during the pulse decay experiment. ${\overline{c_{g}}}_{\text {up }}$ and $\overline{c_{g}}$ down are the mean compressibility for both upstream and downstream reservoirs, respectively. Parameters " $a$ " and " $b$ " are those used in the Dicker and Smits (1988) notation, also given by Eqs. (18) and (19), while " $\gamma$ " and " $\beta$ " are those used in Hsieh et al. (1981) notation for the general solution. The first root of Eq. (15), $\theta_{1}$, was obtained for every pressure equilibration sequence. In addition, the variation of viscosity, gas compressibility and the compressibility factor $\mathrm{Z}$ with pressure are presented in Fig. 4, references in "Appendix 2"sample pseudo-pressure transformation.

The proposed method, which is based on the pseudopressure and pseudo-time transformation, and the $P^{2}$ approach produced similar permeability trends as shown in Fig. 7. Both sets of permeability estimation as a function of pressure show a decrease in permeability as the equilibration pressure increase at a constant boundary stress. Permeability estimation also requires an independent measurement of porosity, which in this case was determined using the Boyle's law, together with the initial and final pressure and the volumes of both upstream and downstream reservoirs in the experimental assembly. The permeability measurements obtained here range between 3.5 and 6 microDarcy, which may appear higher than what is typically measured for shale cores (Tinni et al. 2012) and may correspond with a higher permeability zone. This is attributed to the scale of spatial variations of permeability

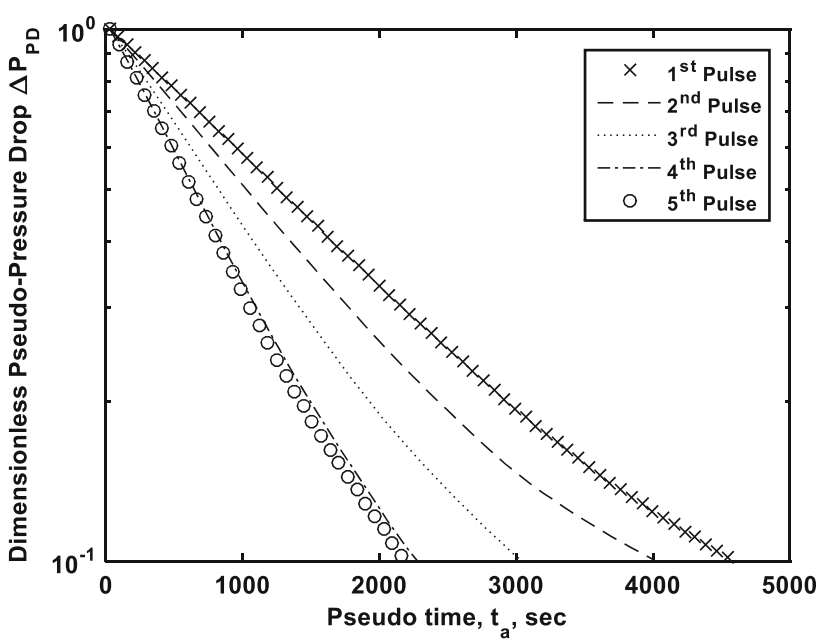

Fig. 5 Dimensionless pseudo-pressure drop as a function of the pseudo-time 


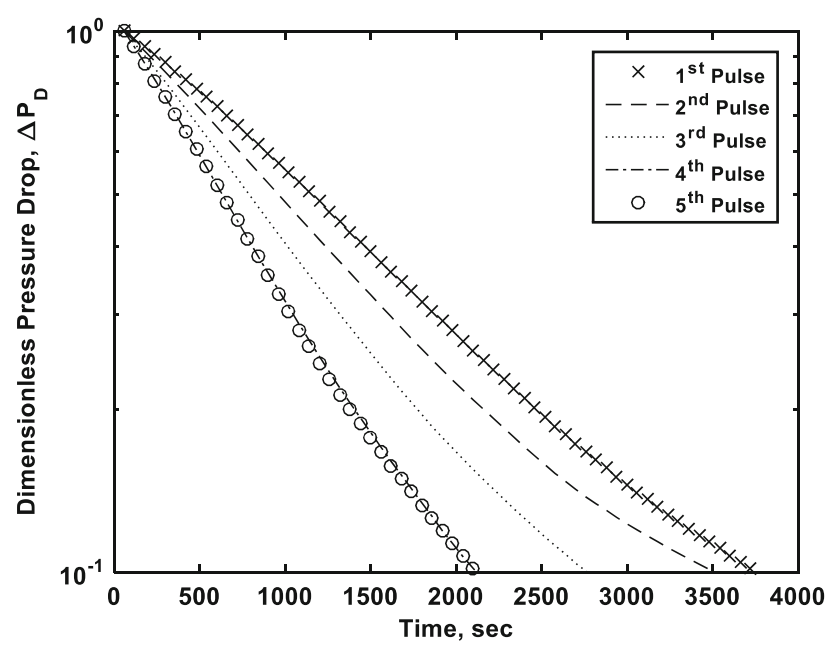

Fig. 6 Dimensionless $P^{2}$ drop as a function of time

within the reservoir, relative to sample size, which may correspond with a higher permeability zone. Porosity measurements obtained through gas expansion porosimetry range 3-9\% with changes in pore pressure and net stress conditions. These results are reported in detail in Abdelmalek (2016).

The similarity in permeability results between the proposed pseudo-pressure and pseudo-time straight-line approach and the $P^{2}$-approach is also evident through the definition of the pseudo-pressure in Eq. (2). At low pressure ranges, the product of viscosity and gas deviation factor $(Z)$ is almost constant; therefore, the integral in Eq. (2) becomes:

$$
\int_{P_{\mathrm{o}}}^{P} \frac{P}{\mu \mathrm{Z}} \mathrm{d} p=\frac{1}{\mu \mathrm{Z}} \int_{P_{\mathrm{o}}}^{P} P \mathrm{~d} p=\frac{1}{\mu \mathrm{Z}} \cdot\left[\frac{1}{2} P^{2}\right]_{P_{\mathrm{o}}}^{P}
$$

With respect to gas slippage during the course of these experiments, it is known from Klinkenberg (1941) that at low pressures, slippage of gas molecules should be expected and that the apparent permeability will be higher than the absolute permeability. Accordingly, permeability was observed to increase for pore/equilibration pressures lower than 400 psi. Above 400 psi, permeability appears to be controlled by pore aperture as reflected by an increase in permeability.

\section{Conclusions}

This work demonstrates the derivation and implementation of an approximate pseudo-pressure versus pseudo-time analysis approach to analyze laboratory pulse decay data and estimate shale permeability of rock cores obtained from a Marcellus shale well. The method has the practical advantage of using the slope of a straight-line to determine the permeability, with the more rigorous treatment of gas properties offered by the pseudo-pressure and pseudo-time transformation of the

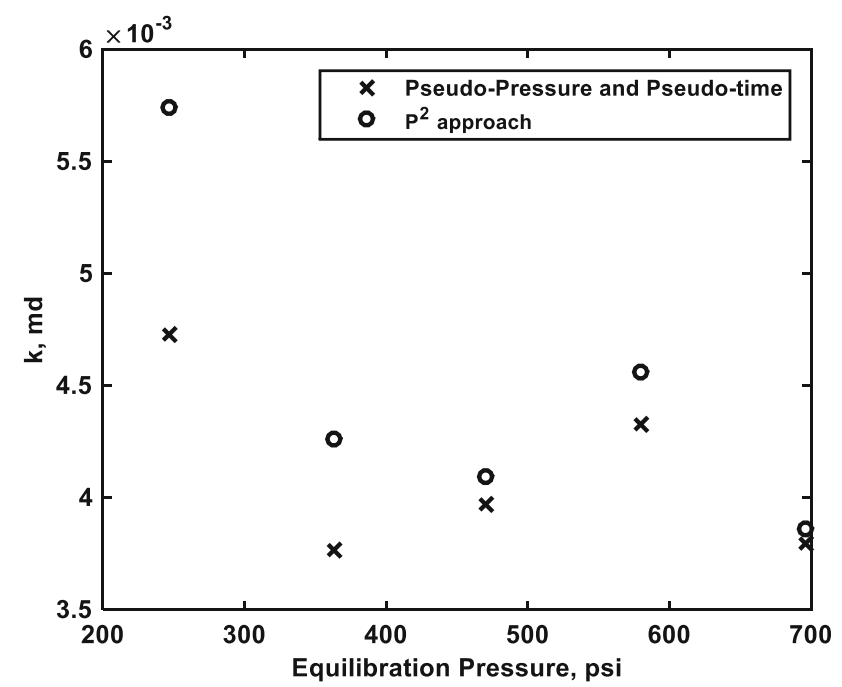

Fig. 7 Permeability calculations using the proposed pseudo-pressure and pseudo-time straight-line approach and the $P^{2}$-approach

compressible flow equation. Experimental results show sensitivity of permeability measurements to pore pressure, which are attributed to a combination of gas flow slippage and pore/ rock compressibility.

Acknowledgements This work received funding support from the US Department of Energy, the Office of Science, Basic Energy Sciences program under Award Number DE-SC0006883. Sandia National Laboratories is a multimission laboratory managed and operated by National Technology and Engineering Solutions of Sandia, LLC., a wholly owned subsidiary of Honeywell International, Inc., for the US Department of Energy's National Nuclear Security Administration under Contract DE-NA-0003525.

Open Access This article is distributed under the terms of the Creative Commons Attribution 4.0 International License (http:// creativecommons.org/licenses/by/4.0/), which permits unrestricted use, distribution, and reproduction in any medium, provided you give appropriate credit to the original author(s) and the source, provide a link to the Creative Commons license, and indicate if changes were made.

\section{Appendix 1: Sample calculation of $\phi_{m}$ roots}

The solution for $\phi_{\mathrm{m}}$ 's (roots of the equation) in this work is going to be obtained numerically using Newton-Raphson technique as follows:

$$
\begin{aligned}
& F=\tan \theta_{m}-\frac{(1+\gamma) \theta_{m}}{\frac{\gamma \theta_{m}^{2}}{\beta}-\beta} \\
& F^{\prime}=\operatorname{Sec}^{2} \theta_{m}-\frac{\left((1+\gamma)\left(\frac{\gamma \theta_{m}^{2}}{\beta}-\beta\right)-\left(\frac{2 \gamma \theta_{m}}{\beta}\right)\left((1+\gamma) \theta_{m}\right)\right)}{\left(\frac{\gamma \theta_{m}^{2}}{\beta}-\beta\right)^{2}}
\end{aligned}
$$


$\theta_{m, \text { new }}=\theta_{m, \text { old }}-\frac{F}{F^{\prime}}$

It should be noted that Eq. (15) has infinite roots. However, one only needs the first two or three positive roots for the solution to work. In order for the numerical scheme to start, one has to make an initial guess for the $\theta_{\mathrm{m}}$ and keep iterating until the error $=\operatorname{abs}\left(\theta_{m, \text { new }}-\theta_{m, \text { old }}\right)$ becomes very small (ex: 0.0001).

for $=1$ and $\beta=0.001 \rightarrow 1$

\begin{tabular}{lllll}
\hline & Initial guesses & $\theta_{1}$ & $\theta_{2}$ & $\theta_{3}$ \\
\hline$\beta=0.001$ & $0 \rightarrow 5$ & 0.044718 & & \\
$\beta=0.003$ & $0 \rightarrow 5$ & 0.07744 & & \\
$\beta=0.01$ & $0 \rightarrow 5$ & 0.141304 & & \\
$\beta=0.03$ & $0 \rightarrow 5$ & 0.244338 & & \\
$\beta=0.1$ & $0 \rightarrow 5$ & 0.443521 & 3.203994 & \\
$\beta=0.3$ & $0 \rightarrow 5$ & 0.755755 & 3.321733 & \\
$\beta=1$ & $1.5 \rightarrow 5$ & 1.306542 & 3.673194 & 6.58462 \\
\hline
\end{tabular}

\section{Appendix 2: Sample pseudo-pressure transformation}

In order to convert the upstream and downstream data to pseudo-pressures, a numerical integration technique may be used like trapezoidal rule to produce a table for pressure values that corresponds to pseudo-pressure values. A simple computer code can then manifest linear interpolation and convert all the upstream and downstream pressures to pseudo-pressures.

An example for the numerical integration of Eq. (2) for two pressure points is shown in following two equations.

$$
\begin{aligned}
P_{\mathrm{p} 1}= & 2\left[\frac{1}{2} *\left(\frac{P_{1}}{\mu_{1} z_{1}}\right) * P_{1}\right] \\
P_{\mathrm{p} 2}= & 2 \\
& \cdot\left[\frac{1}{2} *\left(\frac{P_{1}}{\mu_{1} z_{1}}\right) * P_{1}+\frac{1}{2}\left(\frac{P_{1}}{\mu_{1} z_{1}}+\frac{P_{2}}{\mu_{2} z_{2}}\right) *\left(P_{2}-P_{1}\right)\right]
\end{aligned}
$$

If the trapezoidal rule is applied on the entire pressure range of interest for argon (gas used in the experiments), the figure shows the result.

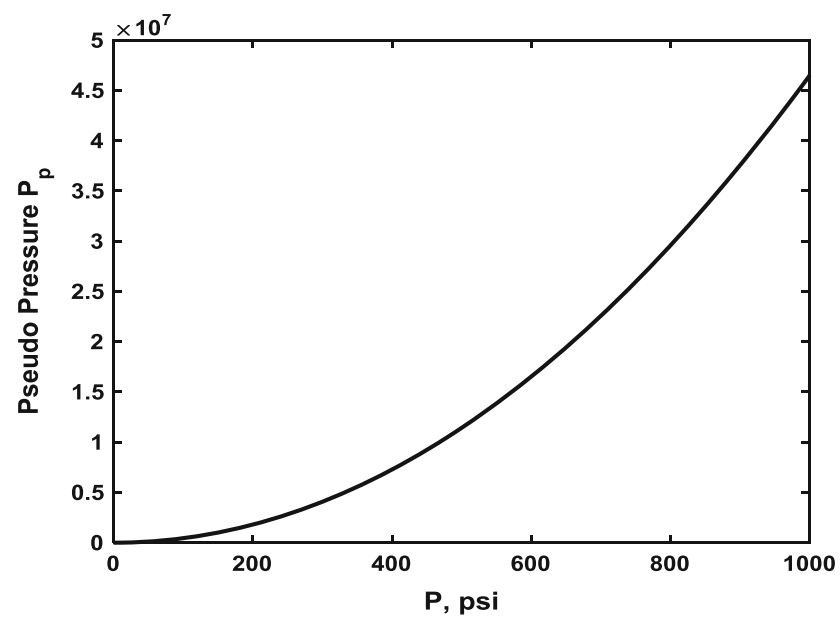

Pseudo-pressure transformation chart

The calculation of fluid properties $c_{\mathrm{g}}, Z$, and $\mu$ required for the pseudo-pressure transformation is presented next.

The Peng-Robinson equation of state is used to obtain the compressibility factor $Z$ :

$\alpha=\left[1+\left(0.37464+1.54226 \omega-0.26992 \omega^{2}\right)\left(1-\sqrt{T_{\mathrm{r}}}\right)\right]^{2}$

$a=0.45724 \frac{R^{2} T_{\mathrm{c}}^{2}}{P_{\mathrm{c}}}$

$b=0.07780 \frac{R T_{\mathrm{c}}}{P_{\mathrm{c}}}$

$A=\frac{\alpha a P}{R^{2} T^{2}}$

$B=\frac{b P}{R T}$

$Z^{3}-(1-B) Z^{2}+\left(A-2 B-3 B^{2}\right) Z-\left(A B-B^{2}-B^{3}\right)$

$$
=0
$$

To obtain gas compressibility $C_{\mathrm{g}}$ :

$c_{\mathrm{g}}=\frac{1}{P}\left[1-\frac{d \ln (z)}{d \ln (p)}\right]$

In order to get it for every pressure point:

$c_{\mathrm{g}, i}=\frac{1}{P_{i}}\left[1-\frac{\left(\ln \left(z_{i-1}\right)-\ln \left(z_{i+1}\right)\right)}{\ln \left(p_{i-1}\right)-\ln \left(p_{i+1}\right)}\right]$ 
The viscosity in this work was obtained from NIST tables for thermodynamics at Temperature $=23{ }^{\circ} \mathrm{C}$.

\section{References}

Abdelmalek BFB (2016) Pseudo-pressure type curve approach for permeability and porosity estimation from pressure-pulse decay data. Master thesis in Energy and Mineral Engineering, The Pennsylvania State University

Boitnott GN (1997) Use of complex pore pressure transients to measure permeability of rocks. In: SPE annual technical conference and exhibition. Society of Petroleum Engineers

Brace WF, Walsh JB, Frangos WT (1968) Permeability of granite under high pressure. J Geophys Res 73:2225-2236

Cui X, Bustin AMM, Bustin RM (2009) Measurements of gas permeability and diffusivity of tight reservoir rocks: different approaches and their applications. Geofluids 9:208-223

Dicker AI, Smits RM (1988) A practical approach for determining permeability from laboratory pressure-pulse decay measurements. In: International meeting on petroleum engineering. Society of Petroleum Engineers

Fischer GJ (1992) Fault mechanics and transport properties of rocks-a festschrift in honor of W. F. Brace Chapter 8 the determination of permeability and storage capacity: pore pressure oscillation method. Int Geophys 51:187-211

Haskett SE, Narahara GM, Holditch SA (1988) A method for simultaneous determination of permeability and porosity in lowpermeability cores. SPE Formation Evaluation
Hsieh PA, Tracy JV, Neuzil CE, Bredehoeft JD, Silliman SE (1981) A transient laboratory method for determining the hydraulic properties of 'tight' rocks-I. Theory. Int J Rock Mech Min Sci Geomech Abstr 18:245-252

Jin G, Pérez HG, Al Dhamen AA, Ali SS, Nair A, Agrawal G, Khodja MR, Hussaini SR, Jangda ZZ, Ali AZ (2015) Permeability measurement of organic-rich shale-comparison of various unsteady-state methods. In: SPE annual technical conference and exhibition. Society of Petroleum Engineers

Jones SC (1997) A technique for faster pulse-decay permeability measurements in tight rocks, SPE Formation Evaluation

Kamath J, Boyer RE, Nakagawa RE (1992) Characterization of core scale heterogeneities using laboratory pressure transients, SPE Formation Evaluation

Klinkenberg L (1941) The permeability of porous media to liquids and gases. In: API 11th mid year meeting. Tulsa

Neuzil CE, Cooley C, Silliman SE, Bredehoeft JD, Hsieh PA (1981) A transient laboratory method for determining the hydraulic properties of 'tight' rocks-II. Application. Int J Rock Mech Min Sci Geomech Abstr 18:253-258

Tinni A, Fathi E, Agarwal R, Sondergeld C, Akkutlu Y, Rai C (2012). Shale permeability measurements on plugs and crushed samples. In: SPE Canadian unconventional resources conference. Society of Petroleum Engineers

Zamirian M, Aminian KK, Ameri S, Fathi E (2014) New steady-state technique for measuring shale core plug permeability. In: SPE/ CSUR unconventional. resources conference-Canada. Society of Petroleum Engineers 